\title{
Reliability of new scores in predicting perioperative mortality after mitral valve surgery
}

\author{
Fabio Barili, MD, PhD, MStat, ${ }^{\mathrm{a}}$ Davide Pacini, MD, ${ }^{\mathrm{b}}$ Claudio Grossi, MD, ${ }^{\mathrm{a}}$ Roberto Di Bartolomeo, MD, \\ Francesco Alamanni, $\mathrm{MD},{ }^{\mathrm{c}}$ and Alessandro Parolari, $\mathrm{MD}, \mathrm{PhD}^{\mathrm{c}}$
}

\begin{abstract}
Objective: The study was designed to validate euroSCORE II and ACEF (age, creatinine, and ejection fraction) scores in patients undergoing isolated or associated mitral valve surgery and compare them with logistic euroSCORE and Society of Thoracic Surgeons scores.
\end{abstract}

\begin{abstract}
Methods: Data on 3441 consecutive patients undergoing isolated or associated mitral valve surgery in a 6-year period were retrieved from 3 prospective institutional databases. Discriminatory power was assessed with the $\mathrm{C}$ index. Calibration was evaluated with calibration curves and associated statistics.

Results: In-hospital mortality was $3.4 \%$. Discriminatory power was uniformly good (for euroSCORE II: area under curve, 0.79; 95\% confidence interval, 0.74-0.84; for logistic euroSCORE: area under the curve, 0.78; 95\% confidence interval, 0.74-0.83; for ACEF: area under the curve, 0.73 ; 95\% confidence interval, 0.69 $0.79)$ but significantly higher in euroSCORE models $(P<.05$ for Delong, bootstrap, Venkatraman methods). Calibration pattern was slightly better for the ACEF score, although related summary statistics (unreliability, Hosmer-Lemeshow test, Spiegelhalter $z$-test for calibration accuracy) were not significant even for euroSCORE II. The euroSCORE II demonstrated a performance similar to Society of Thoracic Surgeons score. Logistic euroSCORE confirmed the progressive trend toward overprediction previously demonstrated in the general cardiac surgical population (summary statistics $P<.05$ ). Analysis of score performances in the surgical group studied showed results comparable to the global population.
\end{abstract}

Conclusions: The euroSCORE II and ACEF scores are good predictors of perioperative mortality in patients undergoing isolated or associated mitral valve surgery, with better discrimination for the first and better calibration for the second. No algorithm seems suitable for risk estimation in mid and high-risk patients. (J Thorac Cardiovasc Surg 2014;147:1008-12)

The estimation of perioperative risk has gained an increasingly important role in cardiac surgery, because perioperative mortality is considered one of the main quality indices of cardiac surgery. Its prediction can lead to better patient understanding of the risks associated with the procedures and may also aid decision-making behavior of clinicians and serve as a guide for hospital oversight to allocate resources efficiently and maximize care for high-risk patients. ${ }^{1}$ Several tools have been developed in recent years, and among them the widest diffusion in the European countries has been reached by additive and logistic euroSCOREs. ${ }^{2}$ These algorithms, however, have been demonstrated to be no more

From the Department of Cardiac Surgery, ${ }^{\text {a }} \mathrm{S}$ Croce Hospital, Cuneo, Italy; the Department of Cardiac Surgery, ${ }^{b}$ University of Bologna, Policlinico S Orsola-Malpighi, Bologna, Italy; and the Department of Cardiovascular Sciences, ${ }^{c}$ University of Milan, Centro Cardiologico Monzino IRCCS, Milan, Italy.

Disclosures: Authors have nothing to disclose with regard to commercial support.

Presented at The 2013 American Association for Thoracic Surgery Mitral Conclave, New York, New York, May 2, 2013.

Received for publication April 3, 2013; revisions received June 12, 2013; accepted for publication June 26, 2013; available ahead of print Aug 29, 2013.

Address for reprints: Fabio Barili, MD, PhD, MStats, Department of Cardiac Surgery, S Croce Hospital, Via M Coppino 26, 12100 Cuneo, Italy (E-mail: fabarili@ libero.

it, barili.f@ospedale.cuneo.it).

0022-5223/\$36.00

Copyright (c) 2014 by The American Association for Thoracic Surgery

http://dx.doi.org/10.1016/j.jtcvs.2013.06.042 than adequate in the modern surgical population, with a high risk of overestimation. ${ }^{3}$ The lack of performance has been especially observed in valve surgery subgroups, because these models were developed and validated in a surgical population composed mainly of those undergoing coronary artery bypass grafting $(\mathrm{CABG}){ }^{4}$

To overcome the performance limitations of older euroSCOREs, an updated version has been recently released, with a similar core of risk factors but a different categorization of operations. ${ }^{5}$ Moreover, a new European score, the ACEF (age, creatinine, and ejection fraction) score, has been recently developed on the basis of a very limited number of risk factors. ${ }^{6}$ Recent validation studies of these new scores on the general population and the aortic valve surgery subgroup have been already published; however, no data are available regarding patients who undergo mitral surgery, a developing field for new technologies. This study was designed to validate the euroSCORE II and ACEF scores in patients undergoing isolated and associated mitral valve surgery and to compare their performance with those of the logistic euroSCORE and Society of Thoracic Surgeons (STS) scores.

\section{MATERIALS AND METHODS}

The study population included all patients who underwent isolated or associated mitral valve surgery from January 2006 to April 2012 


\section{Abbreviations and Acronyms \\ ACEF $=$ age, creatinine, and ejection fraction \\ $\mathrm{AUC}=$ area under the curve \\ $\mathrm{CABG}=$ coronary artery bypass grafting \\ $\mathrm{CI}=$ confidence interval \\ STS $=$ Society of Thoracic Surgeons}

(3441 patients enrolled) within the departments of cardiac surgery of 2 university hospitals and 1 regional hospital. The study population was extracted from a larger database that has been updated to April 2012. ${ }^{7}$ Preoperative and demographic information, operative data, and perioperative mortality and complications for all patients were retrieved from the institutional databases, which are prospectively collected. The institutional review boards approved the data set's use for research. The institutional ethical committees approved the study, and the requirement for informed, written consent was waived on the condition that subjects' identities remained masked. Data from the 3 centers were matched and stored in a dedicated data set.

The scores were tested on the prediction of in-hospital mortality. For the evaluation of the performance, the scores were calculated for each patient in accordance with published guidelines with a dedicated software. The STS score can be applied only to isolated mitral surgery and mitral surgery associated with CABG, so the comparison of the new scores with the STS score was performed only in these subcategories.

The performance of the scores was analyzed with a focus on discrimination power and calibration, as previously described. ${ }^{7,8}$ The discrimination performance indicates the extent to which the model distinguishes between patients who will die or survive in the perioperative period. Discrimination performance was evaluated by constructing receiver operating characteristic curves for each model and calculating the area under the curve (AUC) with $95 \%$ confidence intervals (CIs). An area of 1.0 indicates perfect discrimination power, whereas an area of 0.5 indicates no discrimination of the binary outcome. The comparison among curves was analyzed with Delong, bootstrap, and Venkatraman methods, the first 2 of which compare the AUCs and the last the receiver operating characteristics curves themselves. Another index used to evaluate the predictive abilities was the Somers $D_{x y}$ rank correlation between predicted probabilities and observed responses. When $\mathrm{D}_{\mathrm{xy}}=0$, the model is making a random prediction; when $D_{x y}=1$, the predictions are perfectly discriminating.

Calibration refers to the agreement between observed outcomes and predictions, and it was evaluated by generating calibration plots. The perfectly calibrated predictions stay on the $45^{\circ}$ line, whereas curves below and above the diagonal reflect overestimation and underestimation, respectively. For each model, the comparison of actual slope and intercept with the ideal values of 1 and 0 was performed with the $\mathrm{U}$ statistic (unreliability test) and tested against a $\chi^{2}$ distribution with $2 \mathrm{df}$. For testing whether the calibration curve was ideal, we used even the 1-df Spiegelhalter $z$-test, with its 2-tailed $P$ value for calibration accuracy. Moreover, calibration was tested with Hosmer-Lemeshow goodness-of-fit test, which compares observed with predicted values by decile of predicted probability. The accuracy of the models was also tested by calculating the Brier score (quadratic difference between predicted probability and observed outcome for each patient), an overall performance measure that is 0 when the prediction is perfect.

Two-sided statistics were performed with a significance level of 0.05 . For all analyses the R 2.15.1 software was used (R Foundation for Statistical Computing, Vienna, Austria).

\section{RESULTS}

The mean age of the group was $66.3 \pm 11.8$ years, and $44.1 \%$ were female. Isolated mitral valve surgery was performed in 1239 patients $(36.0 \%), 1$ associated procedure was performed in $1461(42.5 \%)$, and 2 or more associated procedures were performed in $741(21.5 \%)$. CABG was performed in 1023 patients $(29.7 \%)$, without other associated procedures in $613(17.8 \%)$. Other major procedures included aortic valve surgery in 1036 patients $(30.1 \%)$, tricuspid valve surgery in $595(17.3 \%)$, surgery for ascending aorta in $108(3.1 \%)$, and surgery for left ventricular aneurysm in $71(2.1 \%)$. In-hospital mortality was $3.4 \%$ (115 patients). The discriminatory power was good for all algorithms (for euroSCORE II: AUC, 0.79; $95 \%$ CI, 0.74-0.84; for logistic euroSCORE: AUC, 0.78 ; $95 \%$ CI, 0.74-0.83; for ACEF: AUC, 0.73; 95\% CI, 0.69-0.79) but significantly higher for the euroSCORE models $(P<.05$ for Delong, bootstrap, and Venkatraman methods; Table 1). The calibration pattern was better for the ACEF score, with a line closer to the ideal diagonal (Figure 1). In addition, related summary statistics were more favorable for the ACEF score, although thy were not significant even for the euroSCORE II. Logistic euroSCORE confirmed the progressive trend toward overprediction previously demonstrated in the general cardiac surgical population (summary statistics $P<.05$; Table 1 ).

The analysis of scores' performances in isolated and associated mitral valve surgery showed comparable results. In isolated mitral surgery subgroup, the discriminatory power was significantly superior in STS score and euroSCORE algorithms (for STS: AUC, $0.82 ; 95 \%$ CI, 0.75-0.90; for euroSCORE II: AUC, $0.81 ; 95 \%$ CI, 0.75-0.88; for logistic euroSCORE: AUC, 0.82 ; 95\% CI, 0.76-0.89; for ACEF: AUC, $0.76 ; 95 \%$ CI, $0.68-0.83 ; P<.05$ for all comparisons between ACEF and other scores and $P>.05$ for comparisons between STS and euroSCOREs). In associated mitral valve surgery, the superiority of discriminatory performance of euroSCORE models was less marked and not significant (for euroSCORE II: AUC, 0.75 ; 95\% CI, 0.69-0.82; for logistic euroSCORE: AUC, 0.70; 95\% CI, 0.67-0.80; for ACEF: AUC, $0.70 ; 95 \%$ CI, $0.64-0.77 ; P>.05$ for all comparisons). In patients who underwent mitral surgery associated with CABG, the STS score but not euroSCOREs demonstrated a significantly higher discrimination power relative to the ACEF score (for STS score: AUC, 0.76; 95\% CI, 0.65-0.88; for euroSCORE II: AUC, $0.74 ; 95 \%$ CI, 0.61-0.86; for logistic euroSCORE: AUC, $0.73 ; 95 \%$ CI, 0.60-0.85; for ACEF: AUC, 0.70; 95\% CI, 0.56-0.80; $P<.05$ for all comparisons between ACEF and STS score and $P>.05$ for all comparisons between STS and euroSCOREs and between euroSCOREs and ACEF).

The pattern of calibration was similar for all scores in the 2 subgroups. Logistic euroSCORE showed a tendency toward progressive overprediction, which was confirmed even by the associated summary statistics that were significant $(P<.0001$ for unreliability test, Hosmer-Lemeshow test, and Spiegelhalter $z$-test in both isolated and associated 
TABLE 1. Performance of the logistic euroSCORE, euroSCORE II, and ACEF scores in predicting in-hospital mortality among patients undergoing isolated or associated mitral surgery

\begin{tabular}{|c|c|c|c|}
\hline & Logistic ES & ES II & ACEF score \\
\hline Predicted mortality & $8.4 \pm 9.7$ & $3.8 \pm 5.3$ & $3.2 \pm 3.9$ \\
\hline First quartile (25\%) & 2.8 & 1.2 & 1.5 \\
\hline Median value & 5.3 & 2.3 & 1.9 \\
\hline Third quartile $(75 \%)$ & 10.0 & 4.3 & 3.1 \\
\hline \multicolumn{4}{|l|}{ Overall performance } \\
\hline Brier score & 0.03 & 0.03 & 0.03 \\
\hline \multicolumn{4}{|l|}{ Discrimination } \\
\hline AUC $(95 \%$ CI $)$ & $0.78(0.74-0.83)$ & $0.79(0.74-0.84)$ & $0.73(0.69-0.79)$ \\
\hline \multicolumn{4}{|l|}{ DeLong test $P$ value } \\
\hline Versus ACEF & $.02 *$ & $.01 *$ & - \\
\hline Versus ES II & .58 & - & - \\
\hline \multicolumn{4}{|l|}{ Bootstrap method $P$ value } \\
\hline Versus ACEF & $.02 *$ & $.01 *$ & - \\
\hline Versus ES II & .57 & - & - \\
\hline \multicolumn{4}{|l|}{ Venkatraman $P$ value } \\
\hline Versus ACEF & $.04 *$ & $.02 *$ & - \\
\hline Versus ES II & .80 & - & - \\
\hline Somers $\mathrm{D}_{\mathrm{xy}}$ & .57 & .58 & .47 \\
\hline \multicolumn{4}{|l|}{ Calibration } \\
\hline$U$ statistic $P$ value & $.00^{*}$ & .14 & .80 \\
\hline Hosmer-Lemeshow test $P$ value & $.00^{*}$ & .06 & .73 \\
\hline Spiegelhalter $z$-test & $.00 *$ & .10 & .45 \\
\hline
\end{tabular}

Best performance for Brier score $=0$, area under the curve $=1$, Somers Dxy $=1$, nonsignificant $P$ values $(P>.05)$ of the $U$ statistic, Hosmer-Lemeshow test, and Spiegelhalter $z$-test. $E S$, euroSCORE; $A C E F$, age, creatinine, and ejection fraction; $A U C$, area under the curve; $C I$, confidence interval. $* P<.05$.

mitral surgery). On the contrary, even in the subgroups the summary statistics were not significant for euroSCORE II and ACEF scores, although the ACEF score showed a more constant plot with a slight overprediction. In isolated mitral valve surgery and mitral surgery associated with CABG, the STS score demonstrated a calibration pattern similar to that of the euroSCORE II, with nonsignificant associated statistics (isolated mitral surgery unreliability test $P=.47$, Hosmer-Lemeshow test $P=.25$, and Spiegelhalter $z$-test $P=.47$; mitral surgery plus $C A B G$ unreliability test $P=.06$, Hosmer-Lemeshow test $P=.06$, and Spiegelhalter $z$-test $P=.06$ ). In all scores, a tendency toward over miscalibration was evident after $20 \%$ predicted mortality.

Discrimination and calibration of the scores were also tested in patients who underwent elective mitral surgery, because the ACEF score was developed in a nonurgent setting, without finding significant differences with the performance of the scores applied to the entire study group. AUC of ACEF score in elective mitral surgery was 0.71 (95\% CI 0.65-0.77), significantly lower than that of the euroSCORE II and logistic euroSCORE. Even the patterns of calibration plots and the associated statistics were similar when applied to elective surgery and the total population.

\section{DISCUSSION}

The main limitation of the older logistic euroSCORE was confirmed, even in the subgroup of patients who underwent mitral valve surgery, with the tendency toward progressive overprediction evident for both associated and isolated mitral operations and similar to that shown for the general population. ${ }^{7}$ The calibration analysis demonstrated that both the euroSCORE II and ACEF scores have ameliorated the performances of the logistic euroSCORE, as confirmed by the summary statistics. Nonetheless, the patterns of calibration were different. Both scores demonstrated an optimal calibration until $20 \%$ predicted mortality, at which point the plots diverged significantly. The ACEF score in the higher risk showed a better accuracy, with its calibration line closer to the ideal diagonal, although a constant overprediction was still present. The euroSCORE II appeared less calibrated in the higher risk group, with a large fluctuation around the ideal line, and its calibration pattern was similar to that of the STS score in the subgroups for which the latter could be calculated. On the other side, the discriminatory power was higher for the logistic euroSCORE and euroSCORE II, as well as for the STS score, and this outcome is in contrast with previous studies. The ACEF score was previously demonstrated by internal validation to have better global performance than more complex mortality scores, and even a large external validation study demonstrated that the ACEF score has noninferior accuracy and better clinical performance with respect to the additive and logistic euroSCORE. ${ }^{9}$

Risk estimation in cardiac surgery is an evolving issue. Several questions have not been yet addressed, and new advances in techniques and perioperative care are moving 
DISCRIMINATION

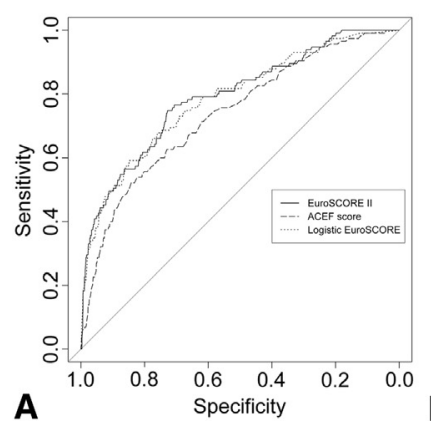

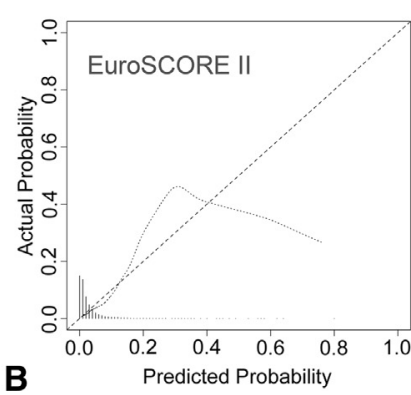

CALIBRATION

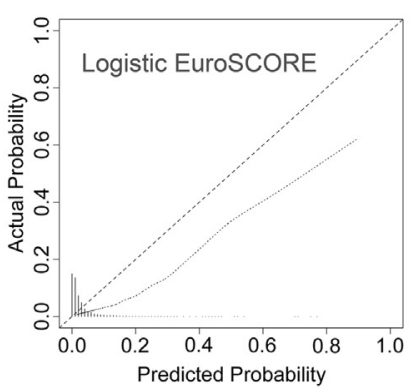

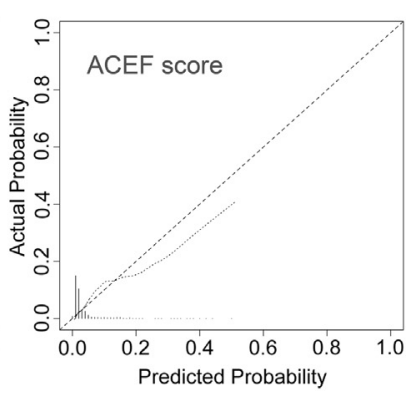

FIGURE 1. Performance evaluation of the ACEF (age, creatinine, and ejection fraction) score, euroSCORE II, and logistic euroSCORE in patients who underwent associated/isolated mitral surgery. A, Receiver operating characteristic curves for the models. B, Calibration plots of the scores are analyzed by means of smooth nonparametric fit with locally weighted scatterplot smoothing. The diagonal lines represent the ideal calibrations.

the attention from the general population toward specific subgroups of patients. The screening and selection of high-risk patients eligible for new surgical techniques, such as transcatheter aortic valve implantation and MitraClip, has underlined a tendency to overpredict the risk of mortality of older euroSCOREs and led to new validation studies confirming the miscalibration. ${ }^{4}$ The lack of calibration of the additive and logistic euroSCOREs has been attributed to the changing epidemiology of cardiac surgery and the nonhomogeneity between the surgical population used to developed the scores and the study groups to which the scores are applied. The new scores have been demonstrated to have better performances in the general surgical population, ${ }^{7}$ and the outcomes of this study confirmed this tendency even for the subgroup of patients who underwent isolated or associated mitral valve surgery. Nonetheless, a common limitation of both the euroSCORE II and ACEF scores remains the tendency toward miscalibration in high-risk deciles. Even in this case, the original study population was mainly composed of those undergoing CABG, and the quota of high-risk patients was small. The use of these scores for the selection of high-risk patients in a specific surgical subgroup thus can lead to lower calibration performance. The suboptimal predictor power in specific subgroups has been already been demonstrated for the STS score as well, even though previous studies have shown that it outperforms the logistic euroSCORE ${ }^{10}$ as it was developed in a larger population and the valvular subgroups are more represented. Nonetheless, the low number of patients with a predicted mortality higher than $15 \%$ leads one to consider cautiously the smooth nonparametric fitted calibration curves in the high-risk patients, because a greater number of higher-risk subjects are requested to obtain more precise estimates, and thus an analysis of score performance in large high-risk patients registries, such as transcatheter valve registries, is necessary to validate euroSCORE II and ACEF scores in high-risk deciles and to confirm the potential miscalibration. Large high-risk groups are also needed to evaluate the role of comorbidities not included in the current score algorithms, such as porcelain aorta, frailty, oxygen-dependent chronic obstructive pulmonary disease, and chest wall deformity. Their inclusion in the models could lead to new dedicated scores that have been already claimed to overcome the potential limitations of existing tools.

\section{Limitations}

The potential limitation of the study is its retrospective nature. Data were derived from three institutional data sets that were prospectively collected. In all data sets, original euroSCORE factors were collected in specific columns together with the scores' values; the logistic euroSCORE for each patient was therefore computed again to check the correctness of the values. The new factors of the euroSCORE II and ACEF scores were derived from the data sets, creatinine clearance was computed as suggested, and categorizations were recalculated from continuous data. Nonetheless, the retrospective nature of scores' calculus can lead to biases if compared with the prospective derivation of the original versions, whose factors were specifically and prospectively collected. Another main limitation of the study is the relative sample size of the study group. Although we collected data from more than 3400 patients who underwent mitral surgery, mortality was $3.4 \%$, with only 115 events, and our results should therefore be confirmed in larger data sets with higher numbers of events. Moreover, $95 \%$ of the sample had a predicted mortality lower than $15 \%$ for both scores, whereas few patients in our study group were at high risk. The pattern of the calibration curves after the first 2 deciles should therefore be confirmed in a high-risk population. Even performance analysis in etiologic subgroups was not possible because of the low number of events.

\section{CONCLUSIONS}

The euroSCORE II and ACEF scores are good predictors of perioperative mortality in patients undergoing isolated or associated mitral valve surgery, with a better discrimination 
for the first and a better calibration for the second. Neither of these algorithms seems suitable for risk estimation in mid and high-risk patients.

\section{References}

1. Sokolovic E, Schmidlin D, Schmid ER, Turina M, Ruef C, Schwenkglenks M, et al. Determinants of costs and resource utilization associated with open heart surgery. Eur Heart J. 2002;23:574-8.

2. Nashef SA, Roques F, Michel P, Gauducheau E, Lemeshow S, Salamon R. European system for cardiac operative risk evaluation (EuroSCORE). Eur J Cardiothorac Surg. 1999;16:9-13.

3. Siregar S, Groenwold RH, de Heer F, Bots ML, van der Graaf Y, van Herwerden LA. Performance of the original EuroSCORE. Eur J Cardiothorac Surg. 2012;41:746-54.

4. Mack MJ. Risk scores for predicting outcomes in valvular heart disease: how useful? Curr Cardiol Rep. 2011;13:107-12.
5. Nashef SA, Roques F, Sharples LD, Nilsson J, Smith C, Goldstone AR, et al. EuroSCORE II. Eur J Cardiothorac Surg. 2012;41:734-44; discussion 744-5.

6. Ranucci M, Castelvecchio S, Menicanti L, Frigiola A, Pelissero G. Risk of assessing mortality risk in elective cardiac operations: age, creatinine, ejection fraction, and the law of parsimony. Circulation. 2009;119:3053-61.

7. Barili F, Pacini D, Capo A, Rasovic O, Grossi C, Alamanni F, et al. Does EuroSCORE II perform better than its original versions? A multicentre validation study. Eur Heart J. 2013;34:22-9.

8. Harrell FE Jr. Regression modelling strategies. New York, NY: Springer; 2001.

9. Ranucci M, Castelvecchio S, Conte M, Megliola G, Speziale G, Fiore F, et al. The easier, the better: age, creatinine, ejection fraction score for operative mortality risk stratification in a series of 29,659 patients undergoing elective cardiac surgery. J Thorac Cardiovasc Surg. 2011;142:581-6.

10. Wendt D, Osswald BR, Kayser K, Thielmann M, Tossios P, Massoudy P, et al. Society of Thoracic Surgeons score is superior to the EuroSCORE determining mortality in high-risk patients undergoing isolated aortic valve replacement. Ann Thorac Surg. 2009;88:468-74; discussion 474-5. 